\title{
Systemic Interleukins Levels in Community: Acquired Pneumonia and their Association with Adverse Outcomes
}

\section{Tripon RE ${ }^{1 *}$, Neagoe IB ${ }^{2}$, Budisan $\mathrm{L}^{2}$, Pop $\mathrm{TL}^{3}$, Cristea V4, Stanca $\mathrm{LM}^{5}$ and Lupse MS ${ }^{1}$}

${ }^{1}$ Department of Infectious Diseases "Iuliu Hatieganu", Teaching Hospital of Infectious Diseases, University of Medicine and Pharmacy, Cluj-Napoca, Romania ${ }^{2}$ Research Center for Functional Genomics, Biomedicine and Translational Medicine "Iuliu Hatieganu" University of Medicine and Pharmacy, Cluj-Napoca, Romania ${ }^{3}$ Department of Pediatrics, "Iuliu Hatieganu", University of Medicine and Pharmacy, Cluj-Napoca, Romania

${ }^{4}$ Department of Immunology, "Iuliu Hatieganu", University of Medicine and Pharmacy, Cluj-Napoca, Romania

${ }^{5}$ Business Information Systems Department, Faculty of Economics and Business Administration, University of Cluj-Napoca, Romania

*Corresponding author: Raluca E Tripon, Department of Infectious Diseases “luliu Hatieganu”, Teaching Hospital of Infectious Diseases, University of Medicine and Pharmacy, Cluj-Napoca, Romania, E-mail: rhorge@yahoo.com; Horge.Raluca@umfcluj.ro

Received October 01, 2018; Accepted October 24, 2018; Published October 31, 2018

Citation: Tripon RE, Neagoe IB, Budisan L, Pop TL, Cristea V, et al. (2018) Systemic Interleukins Levels in Community: Acquired Pneumonia and their Association with Adverse Outcomes. Lung Dis Treat 4: 130. doi:10.4172/2472-1018.1000130

Copyright: @ 2018 Tripon RE, et al. This is an open-access article distributed under the terms of the Creative Commons Attribution License, which permits unrestricted use, distribution, and reproduction in any medium, provided the original author and source are credited.

\section{Retraction Note:}

The article entitled "Systemic Interleukins Levels in Community: Acquired Pneumonia and their Association with Adverse Outcomes" has been accepted for publication in the Journal of Lung Diseases \& Treatment considering the statements provided in the article as personal opinion of the author which was found not having any conflict or biasness towards anything. As the article was a perspective one, information provided by the author was considered as an opinion to be expressed through publication.

Publisher took decision to make the article online solely based on the reviewers suggestion which considered the article not but a personal opinion of the author. However, it is found that the author have some personal concerns and issues, therefore, being retracted from the journal. 